\title{
Beyond Ethylene: New Insights Regarding the Role of AOX in the Respiratory Climacteric
}

\author{
Seanna Hewitt ${ }^{1,2}$ and Amit Dhingra ${ }^{1,2 *}$ \\ 1-Molecular Plant Sciences Graduate Program, Washington State University, Pullman, WA \\ 2-Department of Horticulture, Washington State University, Pullman, WA \\ *Author to whom correspondence should be addressed: adhingra@wsu.edu
}

\begin{abstract}
Climacteric fruits are characterized by a dramatic increase in autocatalytic ethylene production, which is accompanied by a spike in respiration, at the onset of ripening. The change in the mode of ethylene production from autoinhibitory to auto-stimulatory is known as the system 1 (S1) to system 2 (S2) transition. Existing physiological models explain the basic and overarching genetic, hormonal, and transcriptional regulatory mechanisms governing the S1 to S2 transition of climacteric fruit. However, the links between ethylene and respiration, the two main factors that characterize the respiratory climacteric, have been largely understudied at the molecular level. Results of recent studies indicate that the AOX respiratory pathway may play an important role in mediating cross talk between ethylene response, carbon metabolism, ATP production, and ROS signaling during climacteric ripening. New genomic, metabolic, and epigenetic information sheds light on the interconnectedness of ripening-associated metabolic pathways, necessitating expanding the current, ethylene-centric physiological models.

Understanding points at which ripening responses can be manipulated may reveal key, speciesand cultivar-specific targets for regulation of ripening enabling superior strategies for reducing postharvest wastage.
\end{abstract}

Keywords: Ethylene, Ripening, Alternative oxidase, Alternative Respiration, Post-harvest, Phytohormone, System 2 ethylene, Fruit 


\section{Introduction}

Ripening of fruit involves a symphony of transcriptionally and hormonally controlled processes that result in accumulation of sugars, reduction in acidity, development of aroma and nutritional profiles [1]. The ripening process has been under continual manipulation both as a result of natural selection for improved seed dispersal as well as human domestication via selection of desirable organoleptic properties [2, $\underline{3}$ ]. Fleshy fruits fall into one of two broadly defined ripening categories, climacteric and non-climacteric, based on the manner in which they respond to the phytohormone ethylene [4]. Non-climacteric fruit produce ethylene at basal levels throughout development and senescence. This mode of ethylene production is termed System 1 (S1) ethylene production. Non-climacteric fruits, including cherries, berries, and citrus, to name a few, are harvested ripe and do not exhibit increasing levels of ethylene production during ripening, although ripening may be accelerated through exogenous application of ethylene or ethylene-producing compounds such as Ethrel [5-7]. In contrast, ripening in climacteric fruits such as apple, pear, peach, avocado, mango and tomato, is characterized by a substantial increase in ethylene biosynthesis as fruit transitions from S1 to System 2 (S2) ethylene production accompanied by a concomitant burst of respiration (Figure 1) [7, 8]. The synchronization of these two processes is known as the respiratory climacteric. Because of this distinct ripening physiology, climacteric fruit can be harvested unripe, and ripened off the tree or vine [ $\underline{9}, \underline{10}$. Following the respiratory climacteric, ripening proceeds rapidly and irreversibly, which presents additional challenges to the storage and preservation of climacteric fruit after harvest [11]. While the concept of two distinct ripening categories is simple in theory, the reality is far more complex, with certain fruits displaying intermediate phenotypes [12]. Transcriptional and phytohormone regulation of ethylene-dependent ripening have been reviewed extensively [ $1, \underline{7}$, $\underline{13}, 14]$. In contrast to climacteric fruit however, the regulatory network involved in nonclimacteric ripening has been much less studied. Nevertheless, it is known that abscisic acid, rather than ethylene, is essential in the control of ripening in non-climacteric fruits $[\underline{15}, \underline{16}]$. Results of studies in strawberry and tomato suggest that the split between climacteric and nonclimacteric ripening responses may lie in the way that S-adenosyl-L-methionine (SAM) is preferentially utilized as a precursor to ethylene or as a substrate for polyamine biosynthesis, the latter of which is correlated with ABA associated gene expression in non-climacteric systems $[17,18]$. The underlying genetic factors of ripening of both climacteric and non-climacteric fruit have been explored in model systems, laying a foundation for evaluation of ripening processes in fruits exhibiting deviations. Not surprisingly, manipulation of environmental factors, genetic factors, and use of chemical inhibitors like 1-methylcyclopropene (1-MCP) to inhibit ripening result in developmental patterns that don't follow the classical model of ethylene response and signaling [19-22]. Mechanisms for blockage and/or bypass of the concerted steps in classical ethylene biosynthesis are beginning to be elucidated as more studies examine variations in the classical paradigm of ripening via genetic manipulation or exogenous perturbation by temperature or chemicals [23, 24]. As molecular biology, transcriptomics, and epigenetic analysis tools have rapidly advanced, new insights have been gained into some of the master regulators of ripening acting upstream of ethylene [25, 26]. This review briefly revisits the classical model of ripening before exploring points of variation from this model that may result from physiological or chemical perturbations in metabolism, transcriptional regulatory elements, and epigenetic signatures regulating normal ethylene response in fruit. Understanding these 
variations may inform more efficient strategies to reduce postharvest waste while improving marketability of fruit.

\section{Classical model for ethylene-dependent ripening}

Fruits are of great economic and nutritional importance, so the way these plant organs become physiologically and horticulturally mature is of great interest to postharvest biologists. The simple, gaseous, phytohormone ethylene has long been described as the key player in ripening due to its clear effects on fruit organ development, maturation and stress response. Induction of the ripening process may be achieved through both endogenous and exogenous stimulation of ethylene receptors and elicit a number of downstream responses as a result of ethylene signal transduction [1]. The centrality of ethylene to the ripening process has made the components of ethylene biosynthesis and transduction primary targets for ripening control [2, 27]. Tomato (Solanum lycopersicum), is the most extensively studied model system for climacteric fruit ripening as well as for understanding points at which genetic mutations in the ripening pathway result in various non-ripening phenotypes [ $[\underline{2}, \underline{23}]$. The classical model of climacteric ripening in fleshy fruit involves a feed forward cycle of ethylene biosynthesis, signaling, and response. Ethylene is produced endogenously in the plant via the methionine cycle, or Yang cycle [28]. In addition to playing a fundamental role in the biosynthesis of ethylene, the Yang cycle allows for the reutilization of the sulfur-containing amino acid methionine for further biosynthesis of ethylene as well as for other metabolic processes. The first committed step in ethylene biosynthesis is the conversion of L-methionine into S-adenosyl-Lmethionine (SAM). SAM is then converted into 5'-methylthioadenosine (MTA) and 1aminocyclopropane carboxylate (ACC) via ACC synthase (ACS). ACC is subsequently converted into ethylene by 1-aminocyclopropane carboxylate oxidase (ACO). Through a series of steps, MTA is converted back into methionine, allowing for continuation of the cycle without additional input of the amino acid [28]. The catalysis of ethylene production by the enzyme 1aminocyclepropane carboxylate synthase (ACS) is thought to be the rate limiting step in ethylene biosynthesis in fruits, making it a critical point in the S1-S2 transition. While in most fruit, the S1-S2 transition is seamless, European pear (Pyrus communis) requires a pre-ripening period where the fruit is exposed to a genetically pre-determined amount of cold to activate the S2 autocatalytic ethylene biosynthesis [29, 30]. The process of cold temperature exposure is called 'conditioning', which then triggers the fruit to ripen [31] (Figure 1).

The roles of ACS and ACO genes in ethylene biosynthesis and ripening of climacteric fruit, both in model and non-model systems, have been well established; transcripts of both have been shown to increase in expression throughout the ripening climacteric [32, 33]. Following biosynthesis, ethylene is perceived by a family of ER membrane-bound ethylene receptor (ETR) family proteins [7]. Proper ETR function is dependent upon the activity of RAN1, which utilizes a copper ion cofactor, and CONSTITUITIVE-TRIPLE-RESPONSE 1 (CTR1)which mediates transduction of the ethylene signal via ethylene insensitive 2 (EIN2) and ethylene insensitive-like (EIL) family proteins [33-36]. Receptor function has also been shown to involve association with reversion-to-ethylene-sensitivity 1 (RTE1), a negative regulator of ethylene response, and cytochrome b5 proteins [37-39]. Following successful perception of ethylene, the hormone signal is transduced via a series of messengers to the nucleus where ethylene responsive transcription factors activate downstream ripening-associated genes involved in cell wall 
softening, and starch to sugar conversion (Figure 2) [1, $\underline{4}, \underline{40}]$. Recent advances in genome editing have led to questioning of classic understanding of upstream transcriptional regulation of ripening [41], and have provided opportunities for targeted manipulation of genes associated with upstream transcriptional regulation of ripening, fruit texture, photoperiodic response, and post-transcriptional regulation [42]. While ethylene is clearly important in ripening, this pathway does not operate in isolation. Understanding the way that other key pathways may interact with ethylene biosynthesis and response during ripening will lend important insight into how control of ripening in various fruits can be fine-tuned to increase predictability and marketability.

\section{Involvement of $\mathrm{AOX}$ in fruit ripening}

A major aspect of climacteric ripening that has been extensively documented in terms of physiology, but which is often overlooked at the molecular level, is respiration. As respiration has a large impact on postharvest fruit quality correlating directly with senescence, greater examination of the genetic underpinnings of the respiratory climacteric is needed. In climacteric fruits, respiratory rise occurs prior to the S1-S2 ethylene transition. An initially gradual increase in carbon dioxide evolution is followed by a heightened burst in respiratory activity during the ripening climacteric $[\underline{9}, \underline{43}]$.

Climacteric respiration represents the combined activity of several mitochondrial pathways that differentially direct electron transport, leading to several possible energetic fates. The first is the cytochrome c (CYTc) pathway. CYTc operates as a result of a proton gradient generated in the mitochondrial intermembrane space and concludes in the production of cellular energy currency via ATP synthase. In plants, CYTc activity affects the synthesis of antioxidant compounds and influences cellular detoxification [44]. At times when cellular respiratory demands are high, and CYTc is at maximum capacity, the alternative oxidase (AOX) pathway provides a secondary avenue for electron flux. Unlike CYTc, AOX is insensitive to cyanidecontaining compounds, allowing for viability when normal respiratory activity is inhibited [45]. AOX also prevents overreduction of the electron transport chain when electron flux is high through CYTc pathway and can initiate a retrograde message to the nucleus to signal stress in the presence of reactive oxygen species (ROS) [46]. Because of this, AOX activity has been used both as an indicator of stress and as a metric to infer the energetic and metabolic status of plant biological systems during development [47].

Transition from S1-S2 ethylene biosynthesis involves numerous metabolic changes, which may occur simultaneously or in series, that require a great deal of regulation and feedback mechanisms to ensure that ripening occurs properly. There is increasing evidence supporting a role of AOX in the modulation of respiration at various stages around the time of climacteric via induction of S2 ethylene, which thereby influences development of ripening-associated phenotypes downstream [으, 48-50].

\section{Activation of AOX-mediated ripening by external stimuli}

In a number of plant systems, AOX is activated as a result of cold temperatures, a strategy utilized to reduce chilling injury $[\underline{51}, \underline{52}]$. Recently, it has been demonstrated that completion of cold conditioning in European pear, which represents pre-climacteric stage coincided with a maxima in AOX transcript accumulation [30]. Exploiting this natural phenomenon could allow for development of ripening induction strategies in fruits, such as 
European pear that require cold conditioning for ripening. Furthermore, knowledge of how preconditioning with cold temperatures can mitigate chilling injury via AOX stimulation may allow for improved management practices of mango, avocado, banana, zucchini and other temperature sensitive fruits during storage [51-55]. Recent studies provide new insights into the role of AOX in fruit ripening, and how exogenous stimulation (e.g. chemical or temperature) can be utilized to intentionally modulate ripening responses $[\underline{24}, \underline{30}, \underline{56}, 57]$. Results of physiology and gene expression studies of cold conditioned pear fruit suggest that pre-climacteric activity of AOX facilitating the S1-S2 transition directly impacts the ripening process in these fruit [24, 30, 57]. Furthermore, exogenous chemical manipulation of AOX activity with hydrogen sulfide and glyoxylic acid to modulate ripening in pre-climacteric fruit has also been demonstrated [56, $\underline{58}]$. Transcriptomic characterization of expressed genes in response to cold conditioning and to glyoxylic acid application in pear fruit has provided insight into novel ripening-associated pathways and support the putative roles of the AOX alternative respiratory pathway and the glyoxylate cycle in the larger ripening network [24, $\underline{58]}$.

It has been previously demonstrated that organic acids pyruvate and glyoxylic acid directly activate AOX in Arabidopsis through interaction with two cysteine residues that gate the protein [59]. Recent research further elucidates the role of glyoxylic acid in AOX and ripening stimulation through activation of numerous interconnected pathways including: glyoxylate cycle, TCA cycle, fatty acid metabolism, glycolysis, and gluconeogenesis [58]. In tomato, four isoforms of AOX have been identified, with AOX1 isoform expressed in a fruit specific manner $[60,61]$. There is now evidence for activation and differential regulation of AOX protein isoforms and TCA cycle intermediates, as the five homologues present in Arabidopsis demonstrate differential activation as a result of application of TCA cycle metabolites [62]. The results of this study provide information necessary to develop testing "cocktails" of TCA cycle intermediates that could result in optimal activation of alternative respiration in the context of fruit ripening regulation when applied exogenously to pre-climacteric fruit postharvest. Beyond intermediates of primary respiratory metabolism, additional compounds have been shown to enhance the activation of AOX when applied exogenously in appropriate dosages. Hydrogen sulfide $\left(\mathrm{H}_{2} \mathrm{~S}\right)$, though a known phytotoxin, in miniscule doses may serve to enhance alternative pathway respiration and to inhibit ROS production [55, 63-65]. It has also been used as a postharvest processing preservation strategy to reduce oxidative browning and fungal growth in fresh cut sliced pears [66]. Physiological and gene expression studies conducted on 'D'Anjou' and 'Bartlett' pear fruit demonstrated that application of low doses of $\mathrm{H}_{2} \mathrm{~S}$ elicited a pronounced ripening response [56]. In addition to sulfur, AOX has been shown to be induced via the stress hormones salicylic acid (SA) and jasmonic acid (JA) which play a critical role in metabolic adjustments under stress conditions $[\underline{46}, \underline{61}, \underline{67}, \underline{68}]$. Exogenous application of methyl jasmonate and methyl salicylate resulted in increased resistance to chilling injury via activation of AOX $[61,69]$. These findings reveal interesting insights into chemical and hormonal events that operate in an ethylene-independent space during climacteric ripening as well as how ripening can be better regulated as a result of this information.

\section{Molecular and metabolic links between respiration and ethylene}

Clearly, respiration and ethylene are physiologically correlated during climacteric ripening. Pre-climacteric rise in respiration occurs prior to the ethylene spike, but blocking 
ethylene prevents respiration from increasing further. In tomato, 1-MCP treatment reduces transcript levels of AOX1a [49]. The inhibition of ethylene response and maintenance of respiration at low levels by 1-MCP indicates a crosstalk between ethylene and AOX at the molecular level.

Results of several studies point towards direct crosstalk between ethylene, ROS signaling, and alternative respiration in response to stress [70]. Ethylene signaling most often occurs as a stress response. As such, it is accompanied by generation of reactive oxygen species. ROS have historically been thought of in terms of their toxicity to plants in high concentrations, however the critical roles they play in signaling and crosstalk between pathways has become clearer only recently [71]. In Arabidopsis, ethylene has been shown to activate AOX through signaling by hydrogen peroxide $\left(\mathrm{H}_{2} \mathrm{O}_{2}\right)$, a form of ROS, in response to cold temperatures [ 72 , 73]. Conversely, AOX-mediated generation of ROS is thought to be responsible for retrograde signaling to the nucleus communicating the redox state of the mitochondria, thereby eliciting antioxidative responses and alteration in metabolic processes [74]. Cold signals perceived by AOX are likely initiated via activation of membrane bound respiratory burst oxidase (NADPH oxidase) homologs. These NADPH oxidases produce $\mathrm{O}_{2}{ }^{-}$, thereby triggering additional downstream ROS activated processes [75] (Figure 3).

In addition to ROS signaling, activity of AOX and biosynthesis of ethylene are directly dependent upon flux through CYTc and the availability of ATP. RNA interference studies in tomato reveal a modulatory role of $A O X$ in ethylene production, as ACS4 activity in $A O X-R N A i$ plants is significantly lower than in wildtype (WT) plants [49]. Reduced activity of ethylene biosynthetic enzymes when $A O X$ is silenced could be due to a decrease in precursors for ethylene production. For example, the methionine cycle, and therefore ethylene biosynthesis, is dependent upon ATP generation via respiration. Specifically, methionine is converted to the immediate precursor to ACC, S-adenosyl-L-methionine (SAM), in an ATP dependent reaction catalyzed by SAM synthetase [28]. During ripening, AOX may allow for heightened carbon flux through glycolysis and the TCA cycle; prevention of overreduction of the ubiquinone pool in combination with increased carbon turnover results in the production of large amounts of ATP that can be used for S2 ethylene and other ripening-associated metabolic processes. Taken together, these results indicate that the regulatory effects of ethylene and AOX go both ways. AOX produces ATP and elicits retrograde signaling to nucleus via ROS. Ethylene signaling may be responsible for initial AOX activity, but the activity of both thereon out is self-perpetuating by means of an auto stimulatory feedback loop (Figure 3). Furthermore, it is possible that external perturbations resulting in increased AOX activity prior to the $\mathrm{S} 2$ transition, has a vacuum effect by causing under reduction of the mitochondrial electron transport chain, forcing the glycolytic pathway and TCA cycle into action to deliver more reducing power, thereby initiating CYTc respiratory activity. Understanding regulation of the respiratory climacteric and how cross talk between ethylene and $\mathrm{AOX}$ is facilitated may require a look at the transcriptional regulators of these responses.

\section{Transcriptional regulation modulates both ethylene and respiratory responses}

Within the last decade, the importance of transcriptional regulation of ripening response has become more evident. During ripening, signals from upstream transcription factors (which 
may be activated by environmental or intrinsic triggers) facilitate a cascade of downstream signaling activity [1]. In fruits, this signaling activity leads to increased respiration, cell wall softening, and changes in production of pigments, volatiles, starch and sugar content, and phytonutrient metabolite content-these processes are all characteristic of ripening, with respiration and biosynthesis of ethylene particularly relevant to climacteric ripening [10, 14]. Among some of the most important transcriptional regulators during ripening are RIN, CNR, TAG1, TAGL1, FUL1, NOR; all of these are involved in a complex and interconnected regulatory network that ultimately leads to fruit ripening and the aforementioned ripeningassociated qualities (Figure 4). A number of studies examining loss of function of some of the most critical of these transcription factors have led insight into the way in which they serve to activate ripening-associated genes [10].

Because of its resultant complete inhibition of ripening in tomato, the rin mutation has become one of the most iconic ripening-associated mutations in studies of climacteric fruit. Rin mutant tomatoes fail to mature beyond the green-ripe stage and do not exhibit the characteristic ripening climacteric of wild type fruit [76]. Commercial varieties of tomato, heterozygous for the rin mutation have been introduced to the market and have displayed increased shelf life with little noticeable alteration to the desired flavor profile [77]. Chromatin immunoprecipitation studies have revealed several direct targets of RIN, including the ethylene biosynthesizing enzyme 1-aminocyclopropene carboxylate oxidase 4 (ACO4) and the $\alpha$-galacturonase ( $\alpha$-gal), an enzyme-associated with cell wall breakdown and fruit softening [78, 79]. It has been demonstrated recently that $\alpha$-galactosidase and ACO4 genes have RIN protein binding sites (CArG box) in their promoter regions. Furthermore, RIN protein has been shown to bind to sites in the promoter of ACS2 [으. It has also been demonstrated that RIN binding is in concordance with demethylation of these promoter regions [81]. Expression of ACS1 and ACO1 in apple are greatly decreased when expression of various RIN-like MADS-box genes is downregulated or silenced [82]. Bisulfite sequencing studies revealed that binding sites in the promoter regions of known transcriptional targets of RIN were found to be demethylated suggesting that demethylation is necessary for RIN binding and development [83]. Treatment with the methyltransferase inhibitor 5-azacytidine resulted in fruit that ripened prematurely, further lending support to demethylation of binding sites as a trigger for RIN activated ripening [25, $\underline{\text { 83] }}$. While the rin mutant was classically understood as a loss of function mutant, recent work suggests that it is actually a gain-of-function mutant that produces a protein that actively represses ripening [41]. Regardless, it is clear that when RIN is perturbed, ripening does not proceed to completion. Tomato Agamous 1 (TAG1), Tomato Agamous-like 1 (TAGL1), and MADS-box transcription factors Fruitful 1 and 2 can form complexes with RIN. Mutation of these regulatory factors also results in decreased ripening capacity, and transgenic repression of TAGL1 results in fruit with similar non-ripening phenotypes [오, 85]. Colorless non-ripening (CNR) transcription factor is a squamosa promoter binding-like protein, which appears to be necessary for RIN to bind to promoters [ $[\underline{79}, \underline{83}]$. With a hypermethylated, heritable promoter that results in reduced transcriptional activity, CNR is a unique example of an epiallele [86]. Thus, the CNR transcription factor lends evidence for a role of epigenetics in critical developmental transitions such as those that occur during S1-S2 ethylene production and ripening. Another TF among the core set of regulatory elements is the NAC-domain containing protein at the tomato non-ripening (NOR) locus. NOR mutants fail to ripen in a physiologically similar manner to RIN and TAGL1 mutants. NOR acts upstream of ethylene biosynthesis and, like RIN, appears to 
bind to promotor regions of genes involved in ethylene biosynthesis, thereby positively regulating ripening [87] (Figure 4). It is unclear whether RIN and NOR interact with one another to stimulate ripening in conjunction. Considering increasing understanding of its regulatory role in ripening, NOR has been a recent target for improving shelf life in tomato fruit [88].

The availability of many of these mutants in tomato, allows for study of consequences of ripening perturbation at the regulatory level. Many studies have demonstrated the detrimental effects of mutations in these key transcriptional regulators on ethylene production and signaling. Recently, the role of AOX has been investigated in NOR, CNR, and RIN mutant fruit [ $\underline{48}, \underline{49}$, 89]. Interestingly, AOX activity elicits differential effects in these mutants, and expression of RIN, CNR, and the ethylene receptor Never Ripe (NR) has been observed in fruit in which $A O X$ is silenced. When $A O X$ was inhibited via RNA interference, the expression of these transcriptional regulators decreased [49]. This is interesting, as CNR acts upstream of ethylene biosynthesis and the NR receptor acts downstream; this observation suggests that AOX plays a yet uncharacterized role in ripening mediated by transcriptional regulators that affect ethylene biosynthesis, signal transduction, and response $[\underline{3}, \underline{24}, \underline{86}]$.

\section{Epigenetic and epigenomic factors in regulation of ripening}

Epigenetics refers to the heritable modifications of the genome beyond the physical nucleotide sequence, including DNA methylation and modifications to histone proteins, while epigenomics refers to all modifications, regardless of heritability [26]. One of the most commonly studied forms of epigenetic modification is DNA methylation. Methylation status is in constant flux due to the changing environment; therefore, condition specific methylation status may be used to infer stress condition, ripening competency, and developmental progress among other things.

Temperature is known to be a major factor in alteration of methylation status, and conditioning of fruit requiring chilling to ripen, or to avoid chilling injury, could affect the methylation of promoter regions of key ripening related genes and regulatory transcription factors. With more tools for epigenetic and epigenomic analyses available, including bisulfite sequencing and PacBio long read sequencing, new insights are being gained into the impact of epigenetic signatures on development and senescence of fruit. Understanding how the epigenome governs downstream transcriptional regulation and response is critical to better understanding ripening and senescence.

Chemically induced demethylation of tomato fruit using 5-azacytidine results in early ripening of fruit. This finding indicates that alteration of methylation status is one of the first steps in regulation of downstream processes associated with ripening. Recent transcriptomic and gene ontology enrichment analysis of cold conditioned 'D'Anjou' and 'Bartlett' pear fruit suggests that both methylation and chromatin modifications may be important for activation of vernalization-associated genes and ripening-associated transcriptional elements [24]. Interestingly, these two cultivars appear to differentially engage expression of vernalization genes VRN1 and VIN3, which could justify the need for different cold exposure time in different cultivars [24]. Studying the epigenomic status in light of mutations to key transcription factors, mentioned previously, illuminates the way that DNA methylation and histone modifications in genetic regulatory elements serve to modulate certain aspects of ripening early on in development. The breadth of factors that may contribute to alterations in the epigenome is still being elucidated. Beyond abiotic influences, it is possible that some signal from mature seeds is 
originally what signals the onset of ripening progression. This hypothesis is supported by the recent characterization of enzymes responsible for removing epigenetic signatures to DNA or histones, such as the recently characterized DEMETER-like DNA demethylase gene SIDML2 in tomato [25]. These proteins are particularly highly expressed in the locular tissue surrounding mature seeds in fruit.

\section{Opportunities to understand the molecular basis of ripening with omics resources}

The ever-growing fields of genomics, transcriptomics, and epigenomics offer high throughput strategies for extrapolation of important biological function and expression information from large datasets in both pairwise and multifactorial time course experiments [ $\underline{90}$, 91]. Use of such next generation sequencing technologies allows for understanding the array of diverse transcriptional responses, interaction of associated pathways, functional implications of the S1-S2 transition, and interdependent roles of ethylene and respiration in ripening [92] [24, 58]. The relatively new ability to examine plant epigenomes, and most recently the "ripenome" at the level of single nucleotide bases reveals further avenues for understanding epigenetic regulators of the transition from pre-climacteric to climacteric, and the subsequent development of species-specific ripening phenotypes [26]. Such approaches used independently or in conjunction, will facilitate identification of candidate regulators of important ripening and fruit quality-associated characteristics in fruits.

\section{Conclusion}

The interplay of ethylene (biosynthesis, signaling and response) and respiration (CYTc and AOX) have been extensively characterized at the physiological level. Studies conducted within the last several years provide new insights with respect to the connection of these two critical pathways, hallmarks of the respiration climacteric, at the molecular and metabolic level. $A O X$ activity begins to increase during the pre-climacteric phase, prior to the S1-S2 ethylene transition, particularly in the context of cold temperature conditioning or other external stimuli. This activity may be accompanied by the accumulation of ROS as the CYTc pathway capacity reaches maximum. At the onset of S2 ethylene stimulation of both ethylene response and alternative pathway respiration appear to be interdependent, with ethylene biosynthesis requiring ATP generated via respiration and activity of respiratory pathways modulated by ethylene responsive transcription factors in the nucleus, as evidenced by the inhibition of ethylene production and respiration by the ethylene receptor antagonist 1-MCP. The commencement of both ethylene and respiration-associated processes is likely due to upstream transcriptional and epigenetic regulators, as well as the signaling activity of ROS generated during increased respiration. Mutants of master transcriptional regulators in tomato have provided a means for the study of their effects on both ethylene production and respiration during ripening. Furthermore, studies have investigated the effects of temperature and chemical manipulation of AOX with the aim to understand ways in which the timing of ripening can be controlled. AOX and related processes represent an avenue to regulate postharvest ripening. Manipulation of all these ripening control points can enable the development of effective postharvest management strategies in different types of fruits and reduce fruit wastage 


\section{Figures}

\section{Climacteric Ripening}

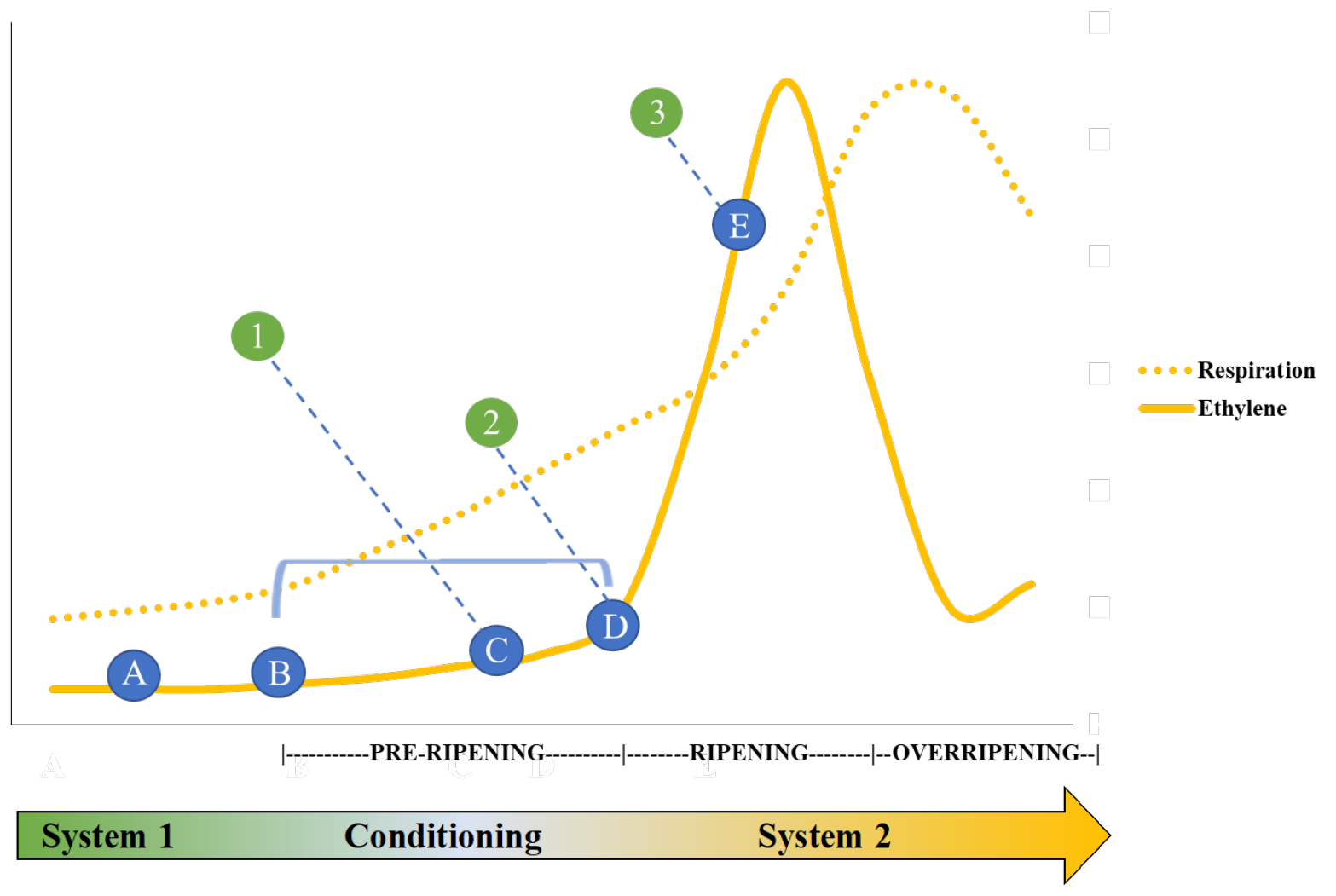

Figure 1. Ripening of climacteric fruit. Numbers refer to degree of responsiveness to ethylene: (1) S1 ethylene production, (2) Start of S2 ethylene production; (3) Increased ethylene response due to autocatalytic synthesis and post-transcriptional effects of ethylene. Letters represent critical points of fruit development: (A) Fruit tissues begin to become sensitive to ethylene; (B) Fruit reaches physiological maturity (seeds are mature); (C) ethylene synthesis reaches level 1; (D) Ethylene synthesis reaches level 2; (E) Ethylene synthesis reaches level 3. Respiration begins to increase during the pre-ripening phase and is amplified greatly after ethylene sensitivity is highest.

Some fruits require pre-climacteric cold conditioning at $0-10^{\circ} \mathrm{C}$ (indicated by the light blue, solid line) to become competent to ripen (Adapted from [31]. 


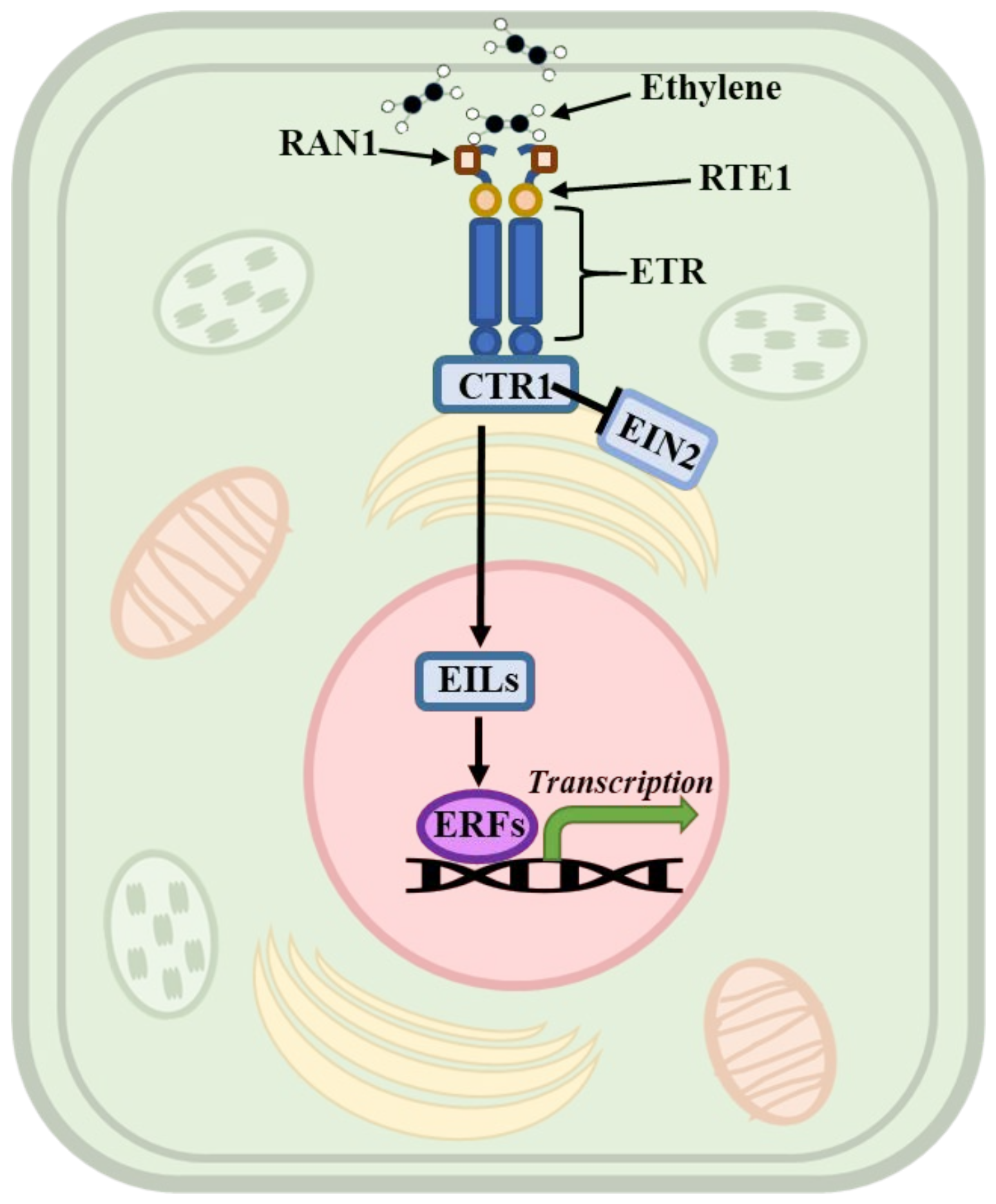

Figure 2. Main components of classical ethylene perception and signaling and cellular location. Ethylene is perceived by ETRs in conjunction with RTE and CTR1. Activation of CTR1 leads to initiation of ethylene responses and inhibition of EIN2, a negative regulator of ethylene signaling. Signal cascades mediated by EILs and ending with translocation of ERFs to the nucleus result in transcriptional activation of ethylene responses. 


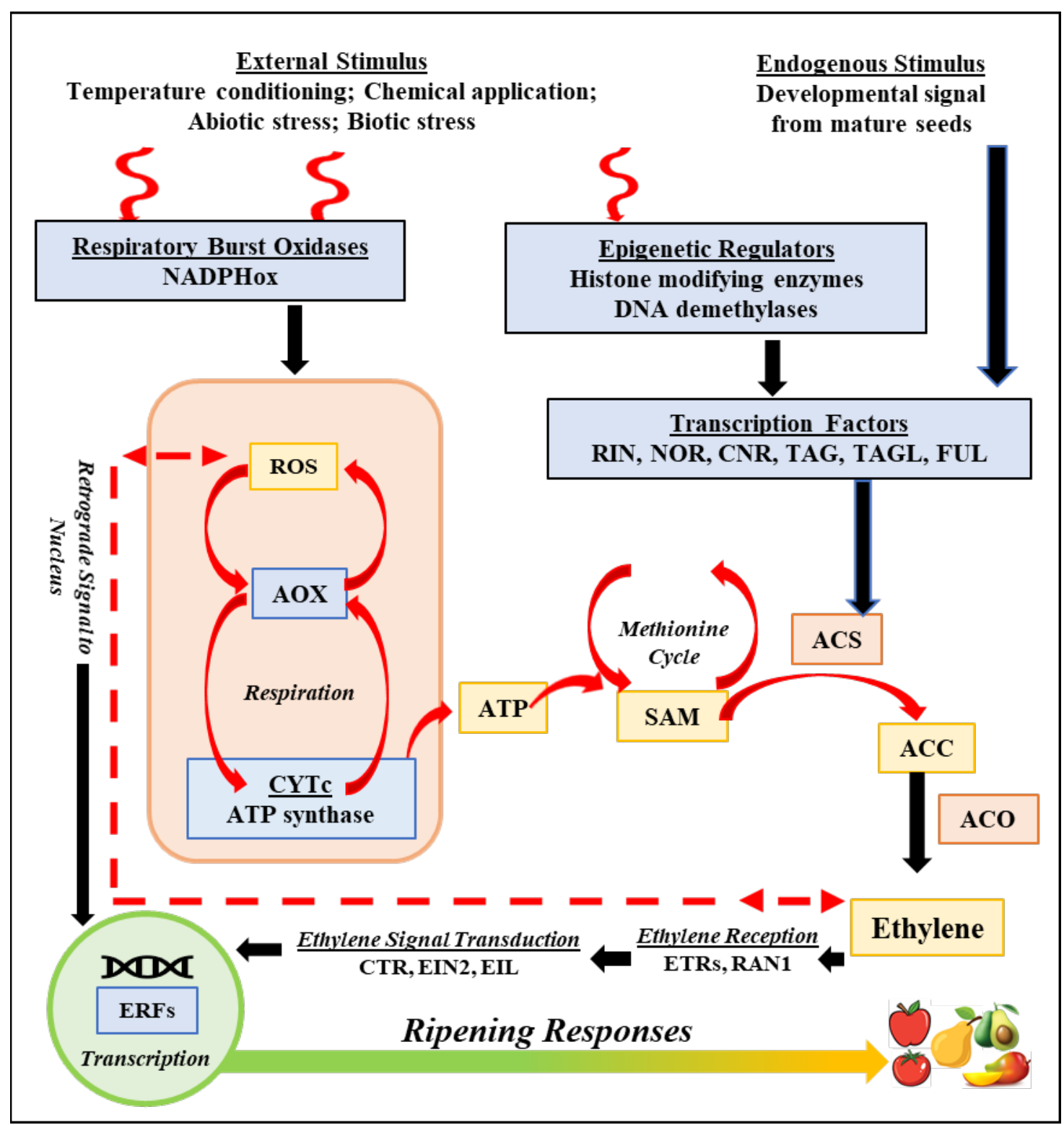

Figure 3. Proposed crosstalk between ethylene and respiration-associated pathways. External stimulus, such as cold or chemical application, induces respiratory burst oxidases and epigenetic regulators of ripening. NADPHox initiates ROS mediated activation of AOX and CYTC pathways. Activation of transcriptional regulators allows for facilitation of downstream ethylene responses. AOX may mediate additional crosstalk with ethylene-associated pathways via retrograde signaling to the nucleus based upon cellular redox state and presence of ROS. 


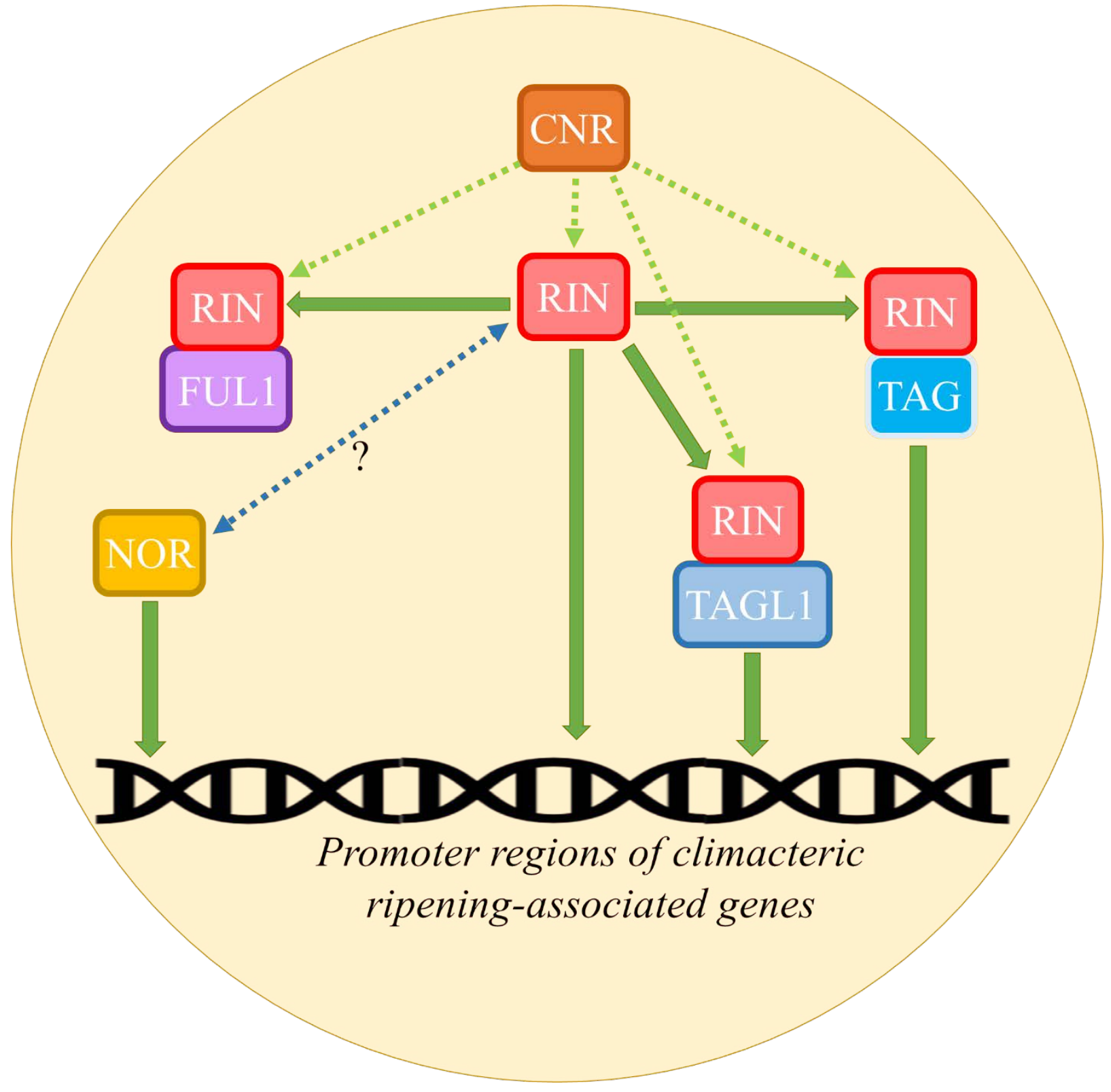

Figure 4. Transcriptional activation of ripening-associated genes. RIN may act independently or in complexes with FUL1, TAG, and TAGL1 transcription factors to activate ripening. Dashed green arrows indicate that direct vs. indirect interaction between RIN and CNR is yet to be determined. Dashed blue arrow indicates that interaction between RIN and NOR is unclear. 


\section{References}

REFERENCES

1. Cherian S, Figueroa CR, Nair H. 'Movers and shakers' in the regulation of fruit ripening: a cross-dissection of climacteric versus non-climacteric fruit. Journal of experimental botany. 2014;65(17):4705-22.

2. Liu M, Pirrello J, Chervin C, Roustan J-P, Bouzayen M. Ethylene Control of Fruit Ripening: Revisiting the Complex Network of Transcriptional Regulation. Plant physiology. 2015;169(4):2380-90. Epub 2015/10/28. doi: 10.1104/pp.15.01361. PubMed PMID: 26511917.

3. Giovannoni JJ. Genetic regulation of fruit development and ripening. The plant cell. 2004;16(suppl 1):S170-S80.

4. Seymour GB, Taylor JE, Tucker GA. Biochemistry of fruit ripening: Springer Science \& Business Media; 2012.

5. Barry CS, Giovannoni JJ. Ethylene and fruit ripening. Journal of Plant Growth Regulation. 2007;26(2):143.

6. Barry CS, Llop-Tous MI, Grierson D. The regulation of 1-aminocyclopropane-1carboxylic acid synthase gene expression during the transition from system-1 to system-2 ethylene synthesis in tomato. Plant Physiology. 2000;123(3):979-86.

7. Chen Y, Grimplet J, David K, Castellarin SD, Terol J, Wong DC, et al. Ethylene receptors and related proteins in climacteric and non-climacteric fruits. Plant science. 2018;276:63-72.

8. Osorio S, Vallarino JG, Szecowka M, Ufaz S, Tzin V, Angelovici R, et al. Alteration of the Interconversion of Pyruvate and Malate in the Plastid or Cytosol of Ripening Tomato Fruit Invokes Diverse Consequences on Sugar But Similar Effects on Cellular Organic Acid, Metabolism, and Transitory Starch Accumulation. Plant Physiology. 2013;161(2):628-43. 
9. Hiwasa-Tanase K, Ezura H. Climacteric and non-climacteric ripening. Fruit Ripening, Physiology, Signalling and Genomics. 2014:1-14.

10. Seymour GB, Chapman NH, Chew BL, Rose JK. Regulation of ripening and opportunities for control in tomato and other fruits. Plant Biotechnology Journal. 2013;11(3):269-78.

11. Jogdand S, Bhat S, Misra K, Kshirsagar A, Lal R. New promising molecules for ethylene management in fruit crops, 1-MCP and nitric oxide: A review. IJCS. 2017;5(3):434-41.

12. Paul V, Pandey R, Srivastava G. The fading distinctions between classical patterns of ripening in climacteric and non-climacteric fruit and the ubiquity of ethylene-An overview2012. 1-21 p.

13. Kumar R, Khurana A, Sharma AK. Role of plant hormones and their interplay in development and ripening of fleshy fruits. Journal of experimental botany. 2014;65(16):4561-75.

14. Karlova R, Chapman N, David K, Angenent GC, Seymour GB, de Maagd RA. Transcriptional control of fleshy fruit development and ripening. Journal of Experimental Botany. 2014;65(16):4527-41.

15. Li C, Jia H, Chai Y, Shen Y. Abscisic acid perception and signaling transduction in strawberry: a model for non-climacteric fruit ripening. Plant Signaling \& Behavior. 2011;6(12):1950-3.

16. Jia H, Jiu S, Zhang C, Wang C, Tariq P, Liu Z, et al. Abscisic acid and sucrose regulate tomato and strawberry fruit ripening through the abscisic acid-stress-ripening transcription factor. Plant biotechnology journal. 2016;14(10):2045-65. Epub 2016/05/04. doi: 10.1111/pbi.12563. PubMed PMID: 27005823. 
17. Guo J, Wang S, Yu X, Dong R, Li Y, Mei X, et al. Polyamines Regulate Strawberry Fruit Ripening by Abscisic Acid, Auxin, and Ethylene. Plant Physiology. 2018;177(1):339-51. doi: 10.1104/pp.18.00245.

18. Van de Poel B, Bulens I, Oppermann Y, Hertog ML, Nicolai BM, Sauter M, et al. Sadenosyl-1-methionine usage during climacteric ripening of tomato in relation to ethylene and polyamine biosynthesis and transmethylation capacity. Physiologia plantarum. 2013;148(2):17688.

19. Watkins CB. Advances in the use of 1-MCP. Advances in postharvest fruit and vegetable technology: CRC Press Boca Raton, FL; 2015. p. 117-45.

20. Watkins CB. The use of 1-methylcyclopropene (1-MCP) on fruits and vegetables. Biotechnology Advances. 2006;24(4):389-409. doi: http://dx.doi.org/10.1016/j.biotechadv.2006.01.005.

21. Tatsuki M, Endo A, Ohkawa H. Influence of time from harvest to 1-MCP treatment on apple fruit quality and expression of genes for ethylene biosynthesis enzymes and ethylene receptors. Postharvest Biol Tec. 2007;43(1):28-35.

22. Chiriboga M-A, Saladié M, Bordonaba JG, Recasens I, Garcia-Mas J, Larrigaudière C. Effect of cold storage and 1-MCP treatment on ethylene perception, signalling and synthesis: Influence on the development of the evergreen behaviour in 'Conference'pears. Postharvest Biol Tec. 2013;86:212-20.

23. Klee HJ, Giovannoni JJ. Genetics and control of tomato fruit ripening and quality attributes. Annual review of genetics. 2011;45:41-59. 
24. Hewitt SL, Hendrickson C, Dhingra A. Evidence for the Involvement of Vernalizationrelated Genes in the Regulation of Cold-induced Ripening in 'D'Anjou' and 'Bartlett' Pear Fruit. bioRxiv. 2019:851733. doi: 10.1101/851733.

25. Liu R, How-Kit A, Stammitti L, Teyssier E, Rolin D, Mortain-Bertrand A, et al. A DEMETER-like DNA demethylase governs tomato fruit ripening. Proceedings of the National Academy of Sciences. 2015;112(34):10804-9.

26. Giovannoni J, Nguyen C, Ampofo B, Zhong S, Fei Z. The epigenome and transcriptional dynamics of fruit ripening. Annual Review of Plant Biology. 2017;68:61-84.

27. Mattoo AK, White WB. Regulation of ethylene biosynthesis. The plant hormone ethylene: CRC Press; 2018. p. 21-42.

28. Yang SF, Hoffman NE. Ethylene biosynthesis and its regulation in higher plants. Annual Review of Plant Physiology. 1984;35(1):155-89.

29. Villalobos-Acuna M, Mitcham EJ. Ripening of European pears: the chilling dilemma. Postharvest Biol Tec. 2008;49(2):187-200. doi: 10.1016/j.postharvbio.2008.03.003. PubMed PMID: WOS:000257364600001.

30. Hendrickson C, Hewitt S, Swanson ME, Einhorn T, Dhingra A. Evidence for preclimacteric activation of AOX transcription during cold-induced conditioning to ripen in European pear (<em>Pyrus communis</em> L.). bioRxiv. 2019:755686. doi: 10.1101/755686.

31. Hartmann C, Drouet A, Morin F. Ethylene and ripening of apple, pear and cherry fruit. Plant Physiology and Biochemistry (France). 1987.

32. Pech J-C, Bouzayen M, Latché A. Climacteric fruit ripening: ethylene-dependent and independent regulation of ripening pathways in melon fruit. Plant Science. 2008;175(1):114-20. 
33. Alexander L, Grierson D. Ethylene biosynthesis and action in tomato: a model for climacteric fruit ripening. Journal of Experimental Botany. 2002;53(377):2039-55.

34. Guo H, Ecker JR. The ethylene signaling pathway: new insights. Current Opinion in Plant Biology. 2004;7(1):40-9. doi: https://doi.org/10.1016/j.pbi.2003.11.011.

35. Binder BM, Rodríguez FI, Bleecker AB. The copper transporter RAN1 is essential for biogenesis of ethylene receptors in Arabidopsis. Journal of Biological Chemistry. 2010;285(48):37263-70.

36. Qiao H, Shen Z, Huang S-sC, Schmitz RJ, Urich MA, Briggs SP, et al. Processing and subcellular trafficking of ER-tethered EIN2 control response to ethylene gas. Science. 2012;338(6105):390-3.

37. Resnick JS, Rivarola M, Chang C. Involvement of RTE1 in conformational changes promoting ETR1 ethylene receptor signaling in Arabidopsis. The Plant Journal. 2008;56(3):42331.

38. Deshpande S, James A, Franklin CH, Leach LJ, Taramonli S, Yang J, editors. An RNASeq Bioinformatics Pipeline for Data Processing of Arabidopsis Thaliana Datasets. Proceedings of the International Conference on Bioinformatics Research and Applications 2017; 2017: ACM. 39. Xu C, Zhou X, Wen C-K. HYPER RECOMBINATION1 of the THO/TREX complex plays a role in controlling transcription of the REVERSION-TO-ETHYLENE SENSITIVITY1 gene in Arabidopsis. PLoS genetics. 2015;11(2):e1004956.

40. Osorio S, Scossa F, Fernie A. Molecular regulation of fruit ripening. Front Plant Sci. 2013;4(198). doi: 10.3389/fpls.2013.00198. 
41. Ito Y, Nishizawa-Yokoi A, Endo M, Mikami M, Shima Y, Nakamura N, et al. Reevaluation of the rin mutation and the role of RIN in the induction of tomato ripening. Nature Plants. 2017;3(11):866-74. doi: 10.1038/s41477-017-0041-5.

42. Martín-Pizarro C, Posé D. Genome Editing as a Tool for Fruit Ripening Manipulation. Front Plant Sci. 2018;9.

43. Colombié S, Beauvoit B, Nazaret C, Bénard C, Vercambre G, Le Gall S, et al. Respiration climacteric in tomato fruits elucidated by constraint-based modelling. New Phytologist. 2017;213(4):1726-39.

44. Welchen E, Gonzalez DH. Cytochrome c, a hub linking energy, redox, stress and signaling pathways in mitochondria and other cell compartments. Physiologia plantarum. 2016;157(3):310-21.

45. Rogov A, Zvyagilskaya R. Physiological role of alternative oxidase (from yeasts to plants). Biochemistry (Moscow). 2015;80(4):400-7.

46. Li CR, Liang DD, Li J, DUAN YB, Li H, YANG YC, et al. Unravelling mitochondrial retrograde regulation in the abiotic stress induction of rice ALTERNATIVE OXIDASE 1 genes. Plant, cell \& environment. 2013;36(4):775-88.

47. Saha B, Borovskii G, Panda SK. Alternative oxidase and plant stress tolerance. Plant signaling \& behavior. 2016;11(12):e1256530.

48. Perotti VE, Moreno AS, Podestá FE. Physiological aspects of fruit ripening: the mitochondrial connection. Mitochondrion. 2014;17:1-6.

49. Xu F, Yuan S, Zhang D-W, Lv X, Lin H-H. The role of alternative oxidase in tomato fruit ripening and its regulatory interaction with ethylene. Journal of Experimental Botany. 2012;63(15):5705-16. doi: 10.1093/jxb/ers226. 
50. Ng S, De Clercq I, Van Aken O, Law SR, Ivanova A, Willems P, et al. Anterograde and retrograde regulation of nuclear genes encoding mitochondrial proteins during growth, development, and stress. Molecular plant. 2014;7(7):1075-93.

51. Carvajal F, Palma F, Jamilena M, Garrido D. Preconditioning treatment induces chilling tolerance in zucchini fruit improving different physiological mechanisms against cold injury. Annals of Applied Biology. 2015;166(2):340-54.

52. Aghdam MS. Role of alternative oxidase in postharvest stress of fruit and vegetables: Chilling injury. African Journal of Biotechnology. 2013;12(51):7009-16.

53. Valenzuela JL, Manzano S, Palma F, Carvajal F, Garrido D, Jamilena M. Oxidative stress associated with chilling injury in immature fruit: postharvest technological and biotechnological solutions. International journal of molecular sciences. 2017;18(7):1467.

54. Lederman IE, Zauberman G, Weksler A, Rot I, Fuchs Y. Ethylene-forming capacity during cold storage and chilling injury development in 'Keitt'mango fruit. Postharvest Biol Tec. 1997;10(1):107-12.

55. Luo Z, Li D, Du R, Mou W. Hydrogen sulfide alleviates chilling injury of banana fruit by enhanced antioxidant system and proline content. Scientia Horticulturae. 2015;183:144-51.

56. Dhingra A, Hendrickson C. Control of ripening and senescence in pre-harvest and postharvest plants and plant materials by manipulating alternative oxidase activity. Google Patents; 2017.

57. Dhingra A, Hendrickson C, Hewitt S, inventors; Google Patents, assignee. Control of ripening and senescence in pre-harvest and post-harvest plants and plant materials2017.

58. Hewitt S, Dhingra A. Glyoxylic acid overcomes 1-MCP induced blockage of fruit ripening in Pyrus communis L. var. ‘D’Anjou. BioRxiv. 2019. 
59. Umbach AL, Ng VS, Siedow JN. Regulation of plant alternative oxidase activity: A tale of two cysteines. Biochimica et Biophysica Acta (BBA) - Bioenergetics. 2006;1757(2):135-42. doi: http://dx.doi.org/10.1016/j.bbabio.2005.12.005.

60. Holtzapffel RC, Finnegan PM, Millar AH, Badger MR, Day DA. Mitochondrial protein expression in tomato fruit during on-vine ripening and cold storage. Functional plant biology. 2002;29(7):827-34.

61. Fung RW, Wang CY, Smith DL, Gross KC, Tao Y, Tian M. Characterization of alternative oxidase (AOX) gene expression in response to methyl salicylate and methyl jasmonate pre-treatment and low temperature in tomatoes. Journal of plant physiology. 2006;163(10):1049-60.

62. Selinski J, Hartmann A, Deckers-Hebestreit G, Day DA, Whelan J, Scheibe R. Alternative oxidase isoforms are differentially activated by tricarboxylic acid cycle intermediates. Plant physiology. 2018;176(2):1423-32.

63. Hu L-Y, Hu S-L, Wu J, Li Y-H, Zheng J-L, Wei Z-J, et al. Hydrogen sulfide prolongs postharvest shelf life of strawberry and plays an antioxidative role in fruits. Journal of agricultural and food chemistry. 2012;60(35):8684-93.

64. Li D, Limwachiranon J, Li L, Du R, Luo Z. Involvement of energy metabolism to chilling tolerance induced by hydrogen sulfide in cold-stored banana fruit. Food chemistry. 2016;208:272-8.

65. Ziogas V, Molassiotis A, Fotopoulos V, Tanou G. Hydrogen sulfide: A potent tool in postharvest fruit biology and possible mechanism of action. Frontiers in Plant Science. 2018;9. 
66. Hu K-D, Wang Q, Hu L-Y, Gao S-P, Wu J, Li Y-H, et al. Hydrogen sulfide prolongs postharvest storage of fresh-cut pears (Pyrus pyrifolia) by alleviation of oxidative damage and inhibition of fungal growth. PloS one. 2014;9(1):e85524.

67. Geigenberger P, Fernie AR. Metabolic control of redox and redox control of metabolism in plants. Antioxidants \& redox signaling. 2014;21(9):1389-421.

68. Gakière B, Hao J, de Bont L, Pétriacq P, Nunes-Nesi A, Fernie AR. NAD+ biosynthesis and signaling in plants. Critical Reviews in Plant Sciences. 2018;37(4):259-307.

69. Wang L, Baldwin EA, Plotto A, Luo W, Raithore S, Yu Z, et al. Effect of methyl salicylate and methyl jasmonate pre-treatment on the volatile profile in tomato fruit subjected to chilling temperature. Postharvest Biol Tec. 2015;108:28-38.

70. Sewelam N, Kazan K, Schenk PM. Global plant stress signaling: reactive oxygen species at the cross-road. Frontiers in plant science. 2016;7:187.

71. El-Maarouf-Bouteau H, Sajjad Y, Bazin J, Langlade N, Cristescu SM, Balzergue S, et al. Reactive oxygen species, abscisic acid and ethylene interact to regulate sunflower seed germination. Plant, cell \& environment. 2015;38(2):364-74.

72. Wang H, Liang X, Huang J, Zhang D, Lu H, Liu Z, et al. Involvement of ethylene and hydrogen peroxide in induction of alternative respiratory pathway in salt-treated Arabidopsis calluses. Plant and Cell Physiology. 2010;51(10):1754-65.

73. Wang H, Huang J, Liang X, Bi Y. Involvement of hydrogen peroxide, calcium, and ethylene in the induction of the alternative pathway in chilling-stressed Arabidopsis callus. Planta. 2012;235(1):53-67.

74. McDonald AE, Vanlerberghe GC. The organization and control of plant mitochondrial metabolism. Annual Plant Reviews online. 2018:290-324. 
75. Suzuki N, Miller G, Morales J, Shulaev V, Torres MA, Mittler R. Respiratory burst oxidases: the engines of ROS signaling. Current opinion in plant biology. 2011;14(6):691-9. 76. Vrebalov J, Ruezinsky D, Padmanabhan V, White R, Medrano D, Drake R, et al. A MADS-box gene necessary for fruit ripening at the tomato ripening-inhibitor (rin) locus. Science. 2002;296(5566):343-6.

77. Garg N, Cheema D, Dhatt A. Utilization of rin, nor, and alc alleles to extend tomato fruit availability. International journal of vegetable science. 2008;14(1):41-54.

78. Fujisawa M, Shima Y, Higuchi N, Nakano T, Koyama Y, Kasumi T, et al. Direct targets of the tomato-ripening regulator RIN identified by transcriptome and chromatin immunoprecipitation analyses. Planta. 2012;235(6):1107-22.

79. Martel C, Vrebalov J, Tafelmeyer P, Giovannoni JJ. The tomato MADS-box transcription factor RIPENING INHIBITOR interacts with promoters involved in numerous ripening processes in a COLORLESS NONRIPENING-dependent manner. Plant physiology. 2011;157(3):1568-79.

80. Fujisawa M, Nakano T, Shima Y, Ito Y. A large-scale identification of direct targets of the tomato MADS box transcription factor RIPENING INHIBITOR reveals the regulation of fruit ripening. The Plant Cell. 2013;25(2):371-86.

81. Li L, Wang X, Zhang X, Guo M, Liu T. Unraveling the target genes of RIN transcription factor during tomato fruit ripening and softening. Journal of the Science of Food and Agriculture. 2017;97(3):991-1000.

82. Ireland HS, Yao JL, Tomes S, Sutherland PW, Nieuwenhuizen N, Gunaseelan K, et al. Apple SEPALLATA1/2-like genes control fruit flesh development and ripening. The Plant Journal. 2013;73(6):1044-56. 
83. Zhong S, Fei Z, Chen Y-R, Zheng Y, Huang M, Vrebalov J, et al. Single-base resolution methylomes of tomato fruit development reveal epigenome modifications associated with ripening. Nat Biotech. 2013;31(2):154-9. doi: 10.1038/nbt.2462

http://www.nature.com/nbt/journal/v31/n2/abs/nbt.2462.html\#supplementary-information.

84. Itkin M, Seybold H, Breitel D, Rogachev I, Meir S, Aharoni A. TOMATO AGAMOUSLIKE 1 is a component of the fruit ripening regulatory network. The Plant Journal. 2009;60(6):1081-95.

85. Garceau DC, Batson MK, Pan IL. Variations on a theme in fruit development: the PLE lineage of MADS-box genes in tomato (TAGL1) and other species. Planta. 2017;246(2):313-21. 86. Seymour GB, Tucker GA, Poole M, Giovann. The molecular biology and biochemistry of fruit ripening: Wiley Online Library; 2013.

87. Gao Y, Wei W, Zhao X, Tan X, Fan Z, Zhang Y, et al. A NAC transcription factor, NOR-like1, is a new positive regulator of tomato fruit ripening. Horticulture research. 2018;5.

88. Nguyen TT, Sim S-C. Development of a Gene-based Marker for the non-ripening (nor) Gene in Cultivated Tomato. 한국원예학회 학술발표요지. 2017:110-.

89. Manning K, Tör M, Poole M, Hong Y, Thompson AJ, King GJ, et al. A naturally occurring epigenetic mutation in a gene encoding an SBP-box transcription factor inhibits tomato fruit ripening. Nature genetics. 2006;38(8):948.

90. Morozova O, Marra MA. Applications of next-generation sequencing technologies in functional genomics. Genomics. 2008;92. doi: 10.1016/j.ygeno.2008.07.001.

91. Banerjee R, Kumar GV, Kumar SJ. Omics-based Approaches in Plant Biotechnology: John Wiley \& Sons; 2019. 
92. Nham NT, Macnish AJ, Zakharov F, Mitcham EJ. 'Bartlett'pear fruit (Pyrus communis L.) ripening regulation by low temperatures involves genes associated with jasmonic acid, cold response, and transcription factors. Plant Science. 2017;260:8-18. 